\title{
Desenvolvimento computacional de um modelo numérico para a análise linear de elementos estruturais da engenharia
}

\author{
Computational development of a numerical model for linear analysis of structural elements \\ of engineering
}

\begin{abstract}
Natan Sian das Neves
Universidade Federal do Rio de Janeiro (UFRJ), Instituto Alberto Luiz Coimbra de Pós-Graduação e Pesquisa de Engenharia (COPPE), Programa de Engenharia Civil (PEC), Ilha do Fundão, RJ, Brasil

iD) https://orcid.org/0000-0002-4679-0461, natan.neves@coppe.ufrj.br
\end{abstract}

\section{Informações do Artigo}

\section{Como citar este artigo}

NEVES, Natan Sian das. Desenvolvimento computacional de um modelo numérico para a análise linear de elementos estruturais da engenharia. REMAT: Revista Eletrônica da Matemática, Bento Gonçalves, RS, v. 7, n. 1, p. e3016, 30 jun. 2021. DOI: https://doi.org/10. 35819/remat2021v7i1id4743

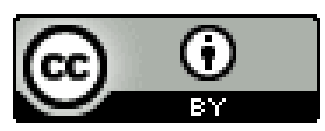

Histórico do Artigo

Submissão: 01 de novembro de 2020.

Aceite: 30 de março de 2021.

Palavras-chave

Elementos Finitos

Estruturas

Código Computacional

Engenharia

Física-Matemática

\section{Resumo}

O presente trabalho concentra-se na aplicação e na apresentação dos aspectos gerais sobre o desenvolvimento do módulo computacional específico para a análise linear de estruturas planas submetidas à ação de cargas externas. Esse módulo faz parte do pacote de solução do programa computacional NASEN. A programação numérica do módulo é concebida de forma estruturada e desenvolvida em ambiente MATLAB. A teoria física desse módulo tem como base os conceitos de elementos estruturais unidimensionais, de placas delgadas e da elasticidade plana. A solução dos modelos estruturais é fundamentada nos procedimentos aproximativos de elementos finitos. Buscando verificar a aplicabilidade do módulo, a investigação numérica é norteada por quatro problemas físicos associados ao comportamento de vigas unidimensionais sob base elástica, de uma placa fina retangular, de uma viga bidimensional engastada-livre e uma chapa tracionada com orifício circular. Os resultados obtidos com o módulo do programa desenvolvido apontam para um comportamento parelho em relação às soluções de referências utilizadas nos casos testes estudados, indicando a precisão e a boa performance do código. 
Keywords

Finite Elements

Structures

Computational Code

Engineering

Physics-Mathematics

\begin{abstract}
The present work focuses on the application and presentation of general aspects about the development of the specific computational module for the linear analysis of plane structures subjected to the action of external loads. This module is part of the solution package of the computer program developed and called NASEN. The numerical programming of the module is designed in a structured way and developed in a MATLAB environment. The physical theory of this module is based on the concepts of one-dimensional structural elements, thin plates and plane elasticity. The solution of the structural models is based on the approximate finite element procedures. Seeking to verify the applicability of the module, the numerical investigation is guided by four physical problems associated with the behavior of one-dimensional beams under elastic base, a thin plate rectangular, a free-clamped two-dimensional beam and a traction plate with a circular hole. The results obtained with the developed program module point to a similar behavior in relation to the reference solutions used in the test cases studied, indicating the precision and good performance of the code.
\end{abstract}

\section{Introdução}

A simulação computacional é uma área que teve um crescimento pronunciado nas últimas décadas devido ao avanço tecnológico. Por consequência dessa evolução constante e da disponibilidade de novos recursos computacionais, torna-se notório uma vasta movimentação de pesquisas que utilizam técnicas numéricas destinadas à solução de diferentes fenômenos físicos presentes na natureza. Esses fenômenos contemplam problemas em inúmeros campos da ciência, como nas áreas da mecânica dos sólidos, termofluidos, acústica, eletromagnetismo, biomédicas, dentre outras. Além disso, os aprimoramentos e as atualizações computacionais acerca da velocidade de processamento, desempenho gráfico e armazenamento de dados, que surgem com o avanço temporal, possibilitam ao meio científico e industrial a solução de diferentes problemas complexos de interesse por meio de métodos numéricos, uma vez que existe capacidade computacional disponível para a simulação (MALISKA, 2017).

As ferramentas de solução de problemas de engenharia podem ser divididas didaticamente em duas classes: os procedimentos teóricos e os experimentais. Os ensaios em laboratórios buscam descrever, em escala real ou reduzida, um problema encontrado na natureza, sendo que esse tipo de procedimento fornece resultados com maior grau de realismo para análise do comportamento do fenômeno físico. Contudo, a ciência experimental enfrenta obstáculos, como o elevado custo financeiro em relação à mão de obra e equipamentos, espaço físico de execução, dificuldades nas 
medições e problemas de escala. Nesta linha, inúmeros estudos científicos são direcionados para utilização de modelos teóricos.

Os métodos analíticos e numéricos compõem, basicamente, a classe dos métodos teóricos. Inicialmente, um modelo matemático visa representar um fenômeno real da natureza, onde na sua construção são utilizados princípios conservativos, leis físicas, relações constitutivas, condições de contorno, dentre outros. A maioria dos problemas físicos de engenharia apresenta um comportamento não trivial, por exemplo, não linearidades do material, imperfeições geométricas, características plásticas, processos transientes, acoplamentos físicos e matemáticos. Sendo assim, como os problemas apresentam diferentes complexidades, recorrentemente na engenharia emprega-se hipóteses simplificadoras de cálculo, dentre elas, pode-se citar, por exemplo, a hipótese de Winkler - que estabelece que o recalque do solo (ou base elástica) é proporcional ao deslocamento, a hipótese de material isotrópico - que afirma que tem as mesmas propriedades independente de qual seja a direção observada (ZIENKIEWICZ; TAYLOR, 2005; TIMOSHENKO, 1979). Além disso, no dimensionamento de estruturas de concreto, normalmente desprezada a pequena resistência à tração do concreto, considerando somente a sua função principal de absorver as tensões de compressão (CARVALHO; FIGUEIREDO FILHO, 2014). Desta maneira, dependendo das aproximações adotadas, esses modelos podem fornecer resultados aceitáveis para predizer a realidade ou simplificações acerca do comportamento do problema, exigindo um aprimoramento dos parâmetros da construção do modelo e, por vezes, a revisão do modelo a fim de obtermos melhores resultados. Além disso, é comum encontrar soluções analíticas somente para problemas específicos, com geometrias e processos físicos simplificados, onde são, frequentemente, utilizados, por exemplo, para calibragem de um programa computacional ou estudo inicial do problema físico.

As soluções aproximadas obtidas por meio de técnicas numéricas são utilizadas como uma alternativa para as soluções analíticas desses problemas. Deste modo, a utilização de métodos de aproximação é uma excelente alternativa para a solução de problemas com maior nível de complexidade, uma vez que existe uma crescente evolução dos dispositivos computacionais, como também nos aprimoramentos dos métodos numéricos utilizados nas soluções. Neste contexto, dentre as técnicas aproximadas usuais na engenharia, como pioneiro, o método das diferenças finitas (MDF) destaca-se pela sua simplicidade e eficiência na solução. A seara computacional ganhou ferramentas robustas de solução por meio do surgimento, em diferentes períodos, do método dos elementos finitos (MEF) e o método dos volumes finitos (MVF), expandindo a solução de problemas em inúmeras áreas da engenharia, como na mecânica dos sólidos e dos fluidos. Com o avanço ma- 
temático e numérico desenvolveram-se otimizações e melhorias, corroborando para os surgimentos de novos métodos, como o método dos elementos de contorno (MEC) e o método meshless.

O comportamento dos elementos estruturais está associado às características do sistema, efeitos e comportamentos, tais como vibrações, grandes deslocamentos e/ou rotações, não linearidades, plasticidade e variações térmicas. Desta maneira, as simulações numéricas buscam representar aproximadamente um modelo físico real, contudo, existem limitações e dificuldades na criação de um modelo computacional com maior verossimilhança possível da realidade (NEVES; SOUZA; PINHEIRO, 2018). Atualmente, no cenário computacional da engenharia estrutural, tem-se programas computacionais comerciais que possuem aplicabilidade em inúmeras áreas de conhecimento, tais como ANSYS, ADINA e ABAQUS, caracterizados pelo investimento preliminar considerável, além de treinamentos e renovações de licenças. Em contrapartida, existem também os programas particulares que são direcionados à solução de problemas específicos, apresentando menor custo inicial. Todavia, eles não possuem uma vasta biblioteca de utilidades para solução de problemas em diferentes áreas físicas, por exemplo, para análises de estruturas sob ação de incêndio cita-se o programa SAFIR (FRANSSEN, 2005), em relação aos problemas da dinâmica de fluidos podese mencionar o programa EasyCFD (LOPES, 2010), para análise térmica bidimensional em regime transiente de estruturas em condições de incêndio cita-se o programa ATERM (PIERIN; SILVA; LA ROVERE, 2015) e para a análise estrutural existe inúmeros programas desenvolvidos, como o FTool (MARTHA, 2002), LESM (RANGEL; MARTHA, 2019), INSANE (PITANGUEIRA et al., 2008) e CSASA (SILVA; PRADO; SILVEIRA, 2013).

Em ambas as escolhas existem vantagens e desvantagens, sendo necessária uma análise do usuário em relação ao problema de interesse e à ferramenta computacional a ser utilizada. Dentro do contexto mencionando, observa-se que devido à demanda de aplicações de problemas ao nível industrial, utilizam-se, rotineiramente, programas comerciais que estão consolidados no mercado, o que garante maior confiabilidade nos resultados. Contudo, pelo fato do usuário não realizar os desenvolvimentos numéricos e matemáticos diretamente nos programas comerciais, deve-se garantir um entendimento básico acerca dos princípios físicos-matemáticos e dos procedimentos gerais de solução adotados no programa, a fim de satisfazer o binômio simulação-segurança.

A implementação computacional baseada na aplicação de técnicas numéricas, como o método dos elementos finitos, baseou-se por muito tempo na programação estruturada realizada em $\mathrm{C}$ ou Fortran. Contudo, novos recursos computacionais estão disponíveis, como a linguagem de alto nível, presentes no MATLAB, Julia e Python, que fornecem, por exemplo, rotinas internas de cálculos 
pré-estabelecidos no sistema e interfaces gráficas iterativas. Sendo assim, as pesquisas sobre implementação autoral buscam aproximar o campo acadêmico-científico com a área industrial, ou seja, os problemas práticos de interesse são encaminhados para os centros de pesquisas ou universidades que visam realizar estudos numéricos, por meio de técnicas e princípios físicos, a fim de compreender o comportamento do elemento em análise. Contudo, em muitas situações, os programas comerciais não possuem pacotes e recursos suficientes para solucionar um determinado problema físico, recorrendo-se ao desenvolvimento de códigos computacionais autorais. Desta maneira, a implementação computacional, além do baixo custo de investimento, possibilita o controle das rotinas numéricas internas do código, sendo que usualmente tais recursos são indisponíveis em programas comerciais e ainda, a longo prazo, é possível a construção de um código computacional eficiente que permite a concatenação de diversas análises de problemas de engenharia.

Neste contexto, apresenta-se neste artigo o sistema computacional denominado NASEN ( $\mathrm{Nu}$ merical Analysis System for Engineering). Esse programa foi desenvolvido em ambiente MATLAB e é dividido em módulos capazes de realizar análises numéricas avançadas de inúmeros problemas clássicos de engenharia. O sistema computacional implementado enquadra-se na solução inicial de problemas para diferentes áreas de conhecimento da engenharia. Até o momento, o programa é dividido em quatro campos físicos de aplicação de interesse para engenharia: área estrutural, térmica, termoestrutural e acústica (ver Figura 1). No trabalho de Neves (2019a), apresenta-se os procedimentos numéricos de solução, a lógica de programação utilizada na implementação dos módulos e a modelagem física e matemática geral dos problemas.

Figura 1 - Áreas físicas e características das análises disponíveis no programa NASEN.

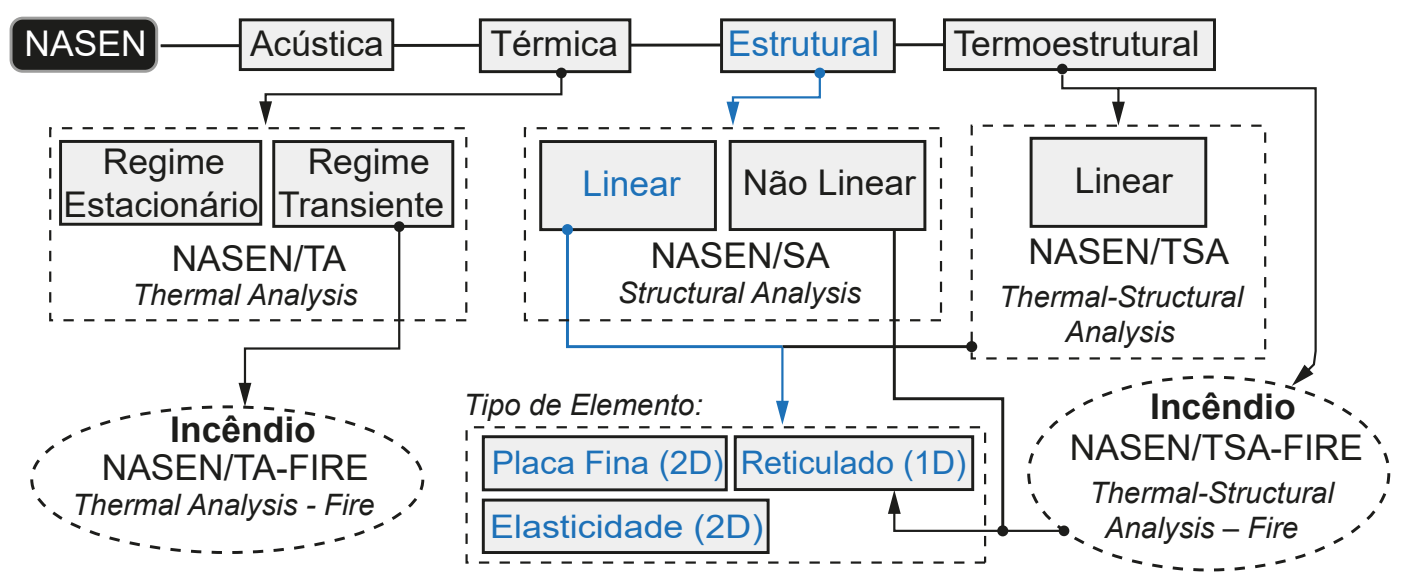

Fonte: Elaboração dos autores (2020). 
O módulo computacional específico NASEN/SA (Structural Analysis) possibilita realizar análises estruturais de natureza linear e não linear. No presente artigo visa-se explorar somente as funcionalidades e capacidades do módulo para a análise linear de estruturas, investigando os problemas descritos pelos modelos lineares com base nas teorias de elementos unidimensionais, de placa delgadas e da elasticidade plana.

\section{Aspectos Físicos-Matemáticos do Modelo Computacional}

O background físico e matemático empregado na construção do módulo computacional específico para análise linear de estruturas, tem como base as formulações de elementos estruturais planos de viga (em inglês, beam), de placa delgada (em inglês, thin plate) e da elasticidade bidimensional. O comportamento físico desses elementos são governados por equações diferenciais (ordinárias ou parciais) com diferentes naturezas, que dependem das características físicas do problema abordado, por exemplo, os efeitos reativos (reação do solo em uma viga de fundação, por exemplo), as não linearidades dos materiais, efeitos dinâmicos e de grandes deslocamentos/rotações. Usualmente, esses modelos são traduzidos matematicamente a partir de três fundamentos básicos da mecânica dos sólidos: as relações de equilíbrio entre forças e tensões, compatibilidade entre deslocamentos e deformações, e as leis constitutivas dos materiais.

Na modelagem matemática, o material apresenta um comportamento elástico-linear, ou seja, a relação tensão-deformação permanece no regime definido pelo limite de proporcionalidade (frequentemente conhecida como lei de Hooke). Para deformações fora desse limite, o comportamento da estrutura torna-se inelástico, devendo ser empregado outro tipo de estratégia de solução nãolinear, conforme pode visto em Ziemian e Mcguire (2002) e Argyris et al. (1982). Além disso, adotase somente os efeitos de primeira ordem, isto é, pode-se escrever as condições de equilíbrio em relação à configuração indeformado da estrutura. Para efeitos de segunda ordem, a solução deve ser direcionada com base em métodos incrementais-iterativos, como pode ser visto em Torkamani, Sonmez e Cao (1997) e Maximiano et al. (2019).

Desta forma, em relação aos elementos planos unidimensionais, o programa NASEN permite estudar o comportamento de vigas, treliça e estruturas aporticadas. Nesta pesquisa, estuda-se, em particular, o comportamento de vigas sob ação de efeitos reativos e de tração. Sendo assim, o elemento de viga é descrito pela equação diferencial de 4⿳â--ordem, caracterizado pela teoria clássica de Euler-Bernoulli, e considerando termos adicionais provenientes da ação do esforço axial de tração 
(T) e os efeitos reativos da base elástica, conforme apresentado na Equação (1):

$$
\frac{d^{2}}{d x^{2}}\left(E I \frac{d^{2} v}{d x^{2}}\right)-\frac{d}{d x}\left(T \frac{d v}{d x}\right)+k_{v} v=q_{y}(x)
$$

onde $E$ é o módulo de elasticidade, $I$ é o momento de inércia, $k_{v}=k_{0} b$, sendo que $k_{0}$ é o um coeficiente de recalque da base elástica e $b$ é a base da viga. Além disso, $v$ e $q_{y}$ são, respectivamente, o campo de deslocamento transversal e o carregamento distribuído ao longo da viga. A teoria Euler-Bernoulli de vigas foi proposta em meados do século XVIII, quando Leonhard Euler expandiu os estudos prévios desenvolvidos por Daniel e Jacob Bernoulli, conforme pode ser visto em Euler (1744). Essa teoria carrega diversas simplificações, dentre elas, destaca-se a negligência dos efeitos dos cisalhantes, da premissa de pequenas deformações e a hipótese de Bernoulli - que afirma que as seções transversais da viga permanecem planas após a deformação.

Para estudar o comportamento de placas delgadas (ou finas) emprega-se a teoria de Kirchhoff. De maneira simplificada, essa teoria apresenta uma semelhança com a teoria de vigas de Euler-Bernoulli, contudo, desenvolvida no âmbito bidimensional. Sendo assim, diferentemente do elemento unidimensional de viga, esse tipo estrutura é governada por uma equação diferencial parcial, definida pela presença do operador biharmônico, conforme posto a seguir:

$$
\frac{\partial^{4} w}{\partial x^{4}}+2 \frac{\partial^{4} w}{\partial x^{2} \partial y^{2}}+\frac{\partial^{4} w}{\partial y^{4}}=\frac{q_{z}(x, y)}{D} ; \quad D=\frac{E h^{3}}{12\left(1-\nu^{2}\right)}
$$

em que $w$ e $q_{z}$ são os campos de deslocamento e do carregamento distribuído na direção $z$, respectivamente, $\nu$ é o coeficiente de Poisson e $h$ é a espessura da placa. Por fim, existem classes de problemas estruturais da engenharia que respeitam certas configurações de geométrica e carregamento que possibilitam ser analisados em um espaço bidimensional, ou seja, grandezas físicas como tensão, deformação e deslocamento dependem somente de duas variáveis espaciais. Neste cenário, tais classes são conhecidas como estado plano de tensão ou deformação. Com base na teoria da elasticidade bidimensional, as equações governantes são apresentadas na Equação (3).

$$
\nabla_{s}^{T} \boldsymbol{\sigma}+\boldsymbol{b}=\mathbf{0} ; \quad \boldsymbol{\sigma}=\boldsymbol{E} \varepsilon \quad \varepsilon=\nabla_{s} \boldsymbol{u}
$$

onde $\nabla_{s}$ é o operador diferencial da relação cinemática, $\sigma$ é o vetor de tensões planas $\left(\sigma_{x x}, \sigma_{y y}\right.$ e $\left.\sigma_{x y}\right), \boldsymbol{b}$ é o vetor de força de campo, $\boldsymbol{u}$ é vetor de deslocamento ( $u$ e $v$ ) e $\boldsymbol{E}$ é a matriz elástica do material. Além disso, na prática da engenharia, é comum definir em projetos critérios de ruptura de elementos estruturais. Sendo assim, considera-se o critério da teoria da energia de distorção máxima aplicável a materiais dúcteis (ou critério de von Mises), a tensão efetiva $\left(\sigma_{v m}\right)$ é expressa matematicamente pela Equação (4) em função das tensões principais $\left(\sigma_{1}, \sigma_{2}\right.$ e $\left.\sigma_{3}\right)$ contidas no 
elemento estrutural.

$$
\boldsymbol{\sigma}_{v m}=\frac{1}{\sqrt{2}}\left[\left(\sigma_{1}-\sigma_{2}\right)^{2}+\left(\sigma_{2}-\sigma_{3}\right)^{2}+\left(\sigma_{3}-\sigma_{1}\right)^{2}\right]^{1 / 2}
$$

Neste contexto, deve-se salientar um ponto importante na concepção desses modelos diferenciais representativos dos comportamentos físicos de elementos estruturais da engenharia, conforme apresentado nas Equações (1)-(4). Sendo assim, a análise de estruturas é fundamentada na construção de modelos matemáticos que representem adequadamente o problema real.

Em diferentes circunstâncias, utiliza-se idealizações estruturais que apresentam grandes aplicabilidades nos problemas de engenharia (WEST, 1989). Para exemplificar esse ponto, a Figura 2 apresenta um croqui puramente didático para mostrar a idealização dos modelos estruturais. Quando realiza-se uma investigação conceitual sobre a concepção dos modelos estruturais é importante considerar a ordem de grandeza das dimensões físicas dos problemas de engenharia. Para um elemento reticulado, a dimensão do eixo da barra é predominante em relação as outras dimensões $(\ell \gg b, h)$. Em contrapartida, para o elemento da elasticidade plana considera-se que a largura da peça real é pequena em relação as demais dimensões $(\ell, h \gg b)$. Por fim, o elemento de placa fina considera que a espessura seja pequena $(\ell, b \gg h)$. Cada elemento apresenta um número de graus de liberdades (GL) diferente em virtude da natureza e comportamento físico das estruturas: (i) elemento de viga (2GL) - uma translação (x, y) e uma rotação (z); (ii) elemento de placa (3GL) - uma translação $(z)$ e duas rotações $(x, y)$ e (iii) elemento da elasticidade (2GL) - duas translações ( $x, y)$.

Figura 2 - Esquema didático simplificado representativo dos modelos estruturais da engenharia.

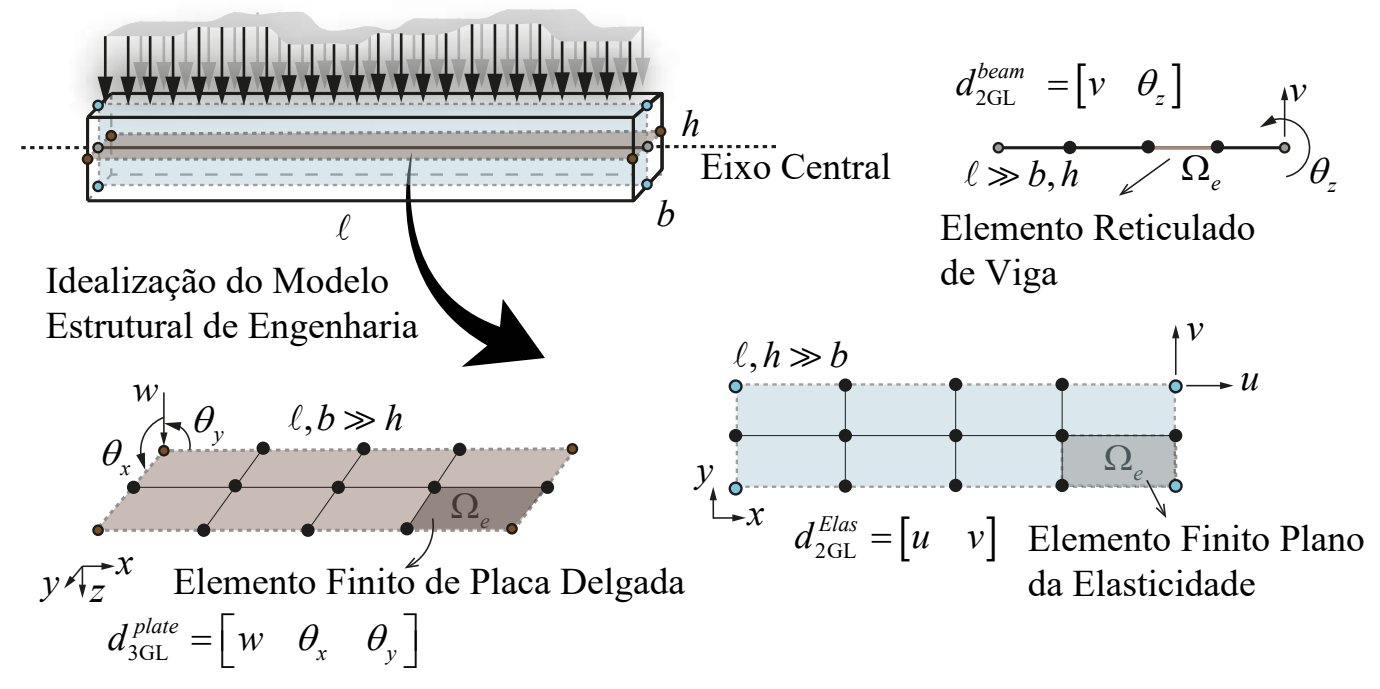

Fonte: Elaboração dos autores (2020). 
Recorrentemente na engenharia estrutural, para solução das equações diferenciais governantes dos problemas físicos, utiliza-se os procedimentos numéricos do método dos elementos finitos (MEF). Contudo, deve-se destacar que esse método é uma extensão natural da técnica de resíduos ponderados, que busca encontrar uma solução aproximada direcionada pela minimização de um resíduo proveniente da equação diferencial (REDDY, 1993). A sentença básica do método é posta na Equação (5),

$$
\int_{\Omega} W_{i} \Re_{\Omega} d \Omega=0 \quad i=1, \ldots, n
$$

onde $W_{i}$ é denominado de função auxiliar (também chamada de função peso ou função teste) e o resíduo é dado por $\Re_{\Omega}=\wp(\hat{u})$, em que $\wp$ é um operador diferencial e $\hat{u}$ é a aproximação da função desconhecida do problema físico. Sendo assim, aplicando a sentença de resíduos nas equações diferenciais governantes dos problemas e com base nos fundamentos do cálculo diferencial-integral (ZIENKIEWICZ; TAYLOR, 2005), chega-se em um sistema algébrico, conforme posto na Equação 6.,

$$
[\boldsymbol{K}]\{\boldsymbol{d}\}=\{\boldsymbol{F}\}
$$

em que $\boldsymbol{K}$ é a matriz de rigidez, $\boldsymbol{d}$ é o vetor de deslocamentos nodais e $\boldsymbol{F}$ é o vetor de forças devido aos carregamentos distribuídos e concentrados atuando nas estruturas. De modo geral, uma das premissas básicas dos procedimentos de cálculo do método do elementos finitos é aproximar o campo de deslocamento geral da estrutura $\left(U^{k}\right)$ como uma combinação linear entre os valores nodais $\left(\bar{U}_{j}\right)$ e as funções de interpolação (ou funções de forma) $N_{j=1,2, \ldots, n}$, sendo $n$ o número de nós dos elementos, conforme apresentado na Equação (8).

$$
U^{k}=\sum_{j=1}^{n} \bar{U}_{j} N_{j}=\bar{U}_{1} N_{1}+\cdots+\bar{U}_{n} N_{n}, \quad k=1,2,3, \ldots
$$

É importante de salientar que $U^{k}$ depende do tipo de modelo estrutural estudado, por exemplo, para elementos da elasticidade plana $(k=2)$, os campos de deslocamento primários são $U^{1}=u$ e $U^{2}=v$. Em contrapartida, para elementos de placas delgadas $(k=1)$, o campo primário é o deslocamento transversal da placa, $U^{1}=w$ (ver Equação (2)). Todavia, é de interesse recorrente da engenharia escrever o deslocamento transversal da placa em função dos deslocamentos $\left(w_{j}\right)$ e das rotações $\left(\theta_{j}\right)$ nodais, introduzindo um elemento de placa com 3 graus de liberdade. Conforme pode ser visto na Figura 2, pode-se generalizar esta ideia de forma matricial, conforme posta a seguir:

$$
\{\mathbf{U}\}=[\boldsymbol{N}]\{\boldsymbol{d}\}
$$

em que $N$ é a matriz geral que contém as funções de forma dos elementos estruturais. Como pode-se ver na Equação (9), cada tipo de elemento estrutural apresenta um vetor de deslocamentos 
nodais diferente, uma vez que os graus de liberdades de interesse são diferentes para cada tipo de modelo.

$$
\begin{aligned}
& \{\boldsymbol{d}\}^{\text {beam }}=\left\{\begin{array}{lllll}
v_{1} & \theta_{z 1} & \ldots & v_{n} & \theta_{z n}
\end{array}\right\}^{T} \\
& \{\boldsymbol{d}\}^{\text {plate }}=\left\{\begin{array}{lllllll}
w_{1} & \theta_{x 1} & \theta_{y 1} & \ldots & w_{2} & \theta_{x 2} & \theta_{y 2}
\end{array}\right\}^{T} \quad\{\boldsymbol{d}\}^{\text {Elas }}=\left\{\begin{array}{llllll}
u_{1} & v_{1} & \ldots & u_{n} & v_{n}
\end{array}\right\}^{T}
\end{aligned}
$$

O procedimento de cálculo da matriz de rigidez de cada elemento segue um padrão de formação, dado por: $[\boldsymbol{K}]=\int_{V}[\boldsymbol{B}]^{T}[\boldsymbol{D}][\boldsymbol{B}] d V$, onde a matriz $\boldsymbol{B}$ é obtida pelas relações cinemáticas e a matriz $\boldsymbol{D}$ associada ao comportamento elástico do material. Desta forma, cada modelo estrutural da engenharia apresenta uma formulação particular para essas matrizes associadas aos comportamentos físicos de cada problema (NEVES, 2019b). Sendo assim, primeiramente, a matriz elementar de rigidez do modelo unidimensional de viga é composto pela superposição do comportamento axial de barras e de flexão de vigas, conforme posto na Equação (10),

$$
\boldsymbol{K}_{\text {beam }}^{e}=k_{i j}=\int_{L}\left[\frac{\partial^{2} N_{i}^{2}}{\partial x^{2}}\right]^{T}[E I]\left[\frac{\partial^{2} N_{j}^{2}}{\partial x^{2}}\right] d x+\int_{L}\left[\frac{\partial N_{i}^{2}}{\partial x}\right]^{T}[T]\left[\frac{\partial N_{j}^{2}}{\partial x}\right] d x+\int_{L}\left[N_{i}^{2}\right]^{T}\left[k_{v}\right]\left[N_{j}^{2}\right] d x
$$

em que EI é a rigidez de flexão. O elemento de placa fina de Kirchhoff apresenta uma matriz cinemática semelhante ao comportamento de vigas de Euler, todavia, no contexto bidimensional, conforme mostra a Equação 11.

$$
\boldsymbol{K}_{\text {plate }}^{e}=k_{i j}=\frac{E}{1-\nu^{2}} \iint_{A}\left[\begin{array}{c}
\frac{\partial^{2} N_{i}^{3}}{\partial x^{2}} \\
\frac{\partial^{2} N_{i}^{3}}{\partial y^{2}} \\
\frac{\partial^{2} N_{i}^{3}}{\partial x \partial y}
\end{array}\right]^{T}\left[\begin{array}{ccc}
1 & \nu & 0 \\
\nu & 1 & 0 \\
0 & 0 & (1-\nu) / 2
\end{array}\right]\left[\begin{array}{c}
\frac{\partial^{2} N_{j}^{3}}{\partial x^{2}} \\
\frac{\partial^{2} N_{j}^{3}}{\partial y^{2}} \\
\frac{\partial^{2} N_{j}^{3}}{\partial x \partial y}
\end{array}\right] d A
$$

Por fim, a matriz de rigidez de um elemento plano da elasticidade segue a Equação (12), válida para o estado plano de tensão (EPT),

$$
K_{\text {elas }}^{e}=k_{i j}=b \iint_{A}\left[\begin{array}{cc}
\frac{\partial N_{i}^{4}}{\partial x} & 0 \\
0 & \frac{\partial N_{i}^{4}}{\partial y} \\
\frac{\partial N_{i}^{4}}{\partial y} & \frac{\partial N_{i}^{4}}{\partial x}
\end{array}\right]^{T} \frac{E}{1-\nu^{2}}\left[\begin{array}{ccc}
1 & \nu & 0 \\
\nu & 1 & 0 \\
0 & 0 & (1-\nu) / 2
\end{array}\right]\left[\begin{array}{cc}
\frac{\partial N_{j}^{4}}{\partial x} & 0 \\
0 & \frac{\partial N_{j}^{4}}{\partial y} \\
\frac{\partial N_{j}^{4}}{\partial y} & \frac{\partial N_{j}^{4}}{\partial x}
\end{array}\right] d A
$$

onde $N^{2}, N^{3}$ e $N^{4}$ são, respectivamente, as funções de interpolação de flexão de vigas, de placas finas e da elasticidade plana. De acordo com a matriz de rigidez local $\left(K^{e}\right)$ das estruturas analisadas, a matriz cinemática $(\boldsymbol{B})$ de cada tipo de elemento segue um padrão de formação, conforme pode ser visto nas Equações (10)-(12). Vale destacar também que as integrais presentes na construção das matrizes de rigidez local, usualmente, são computadas por meio de integração numérica de Gauss. Em alguns casos específicos, dependendo da escolha da função de interpolação, pode-se empregar a integração analítica, conforme pode ser visto na literatura (REDDY, 1993). 


\section{Implementação Computacional}

O módulo específico, NASEN/SA, foi desenvolvido em ambiente MATLAB (2010) devido ser uma linguagem de programação de alto nível e com um boa produtividade no decorrer da implementação, permitindo usar funções e rotinas avançadas predefinidas, como solução de sistemas lineares, cálculo simbólico, dentre outras funcionalidades. Convém enfatizar que tal ferramenta ainda proporciona uma facilidade na visualização dos resultados de problemas de engenharia devido ao conjunto de ambientes gráficos didáticos. A implementação computacional do módulo para análise linear de estruturas do programa NASEN é realizada com base em uma programação estruturada. Sendo assim, a Figura 3 apresenta um fluxograma geral dos principais aspectos e lógica utilizada no desenvolvimento do código.

Figura 3 - Estrutura geral de programação do código computacional para análise linear de estruturas.

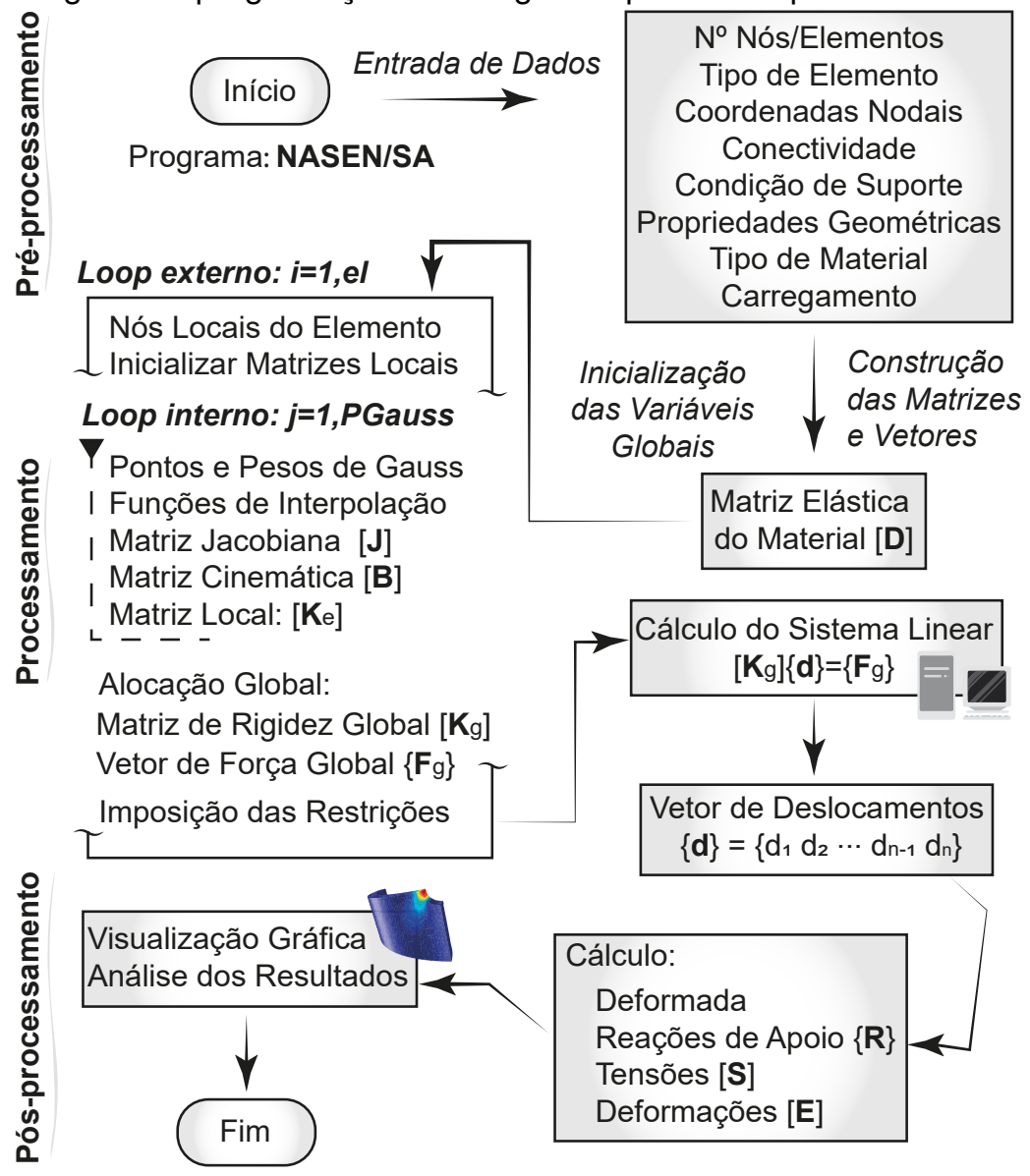

Fonte: Elaboração dos autores (2020).

A estrutura geral de uma simulação computacional de um problema físico da engenharia transita por três pilares: pré-processamento, processamento e pós-processamento. Na etapa inicial, 
o programa necessita da leitura de arquivos de entrada, contendo informações preliminares sobre as estruturas, como os nós e elementos da malha, conectividade, tipo de elemento, condições de apoio e dentre outras informações. A geração de malha bidimensional do programa é auxiliada pelo software free GMSH (GEUZAINE; REMACLE, 2009).

$\mathrm{Na}$ etapa seguinte, realiza-se os cálculos e os procedimentos numéricos indicados para a resolução do problema, como a montagem das matrizes/vetores, integrações numéricas e a solução do sistema linear. Por fim, após obter a resposta primal do problema, realiza-se os cálculos secundários, como reações de apoio, tensões e deformações. Nessa última fase utiliza-se diversos recursos gráficos para auxiliar na investigação do comportamento físico. No programa NASEN, o pós-processamento é realizado com base nos ambientes gráficos iterativos do MATLAB.

\section{Exemplos Numéricos de Aplicação}

A experimentação numérica tem como base a investigação de problemas físicos de natureza estrutural descritos pelos elementos reticulados, de placa e da elasticidade. Para avaliação de desempenho do programa NASEN, utiliza-se a métrica de erro percentual $(\Delta)$ a seguir:

$$
\left|\Delta_{a}^{b}\right|(\%)=100 \times\left|X_{\text {num }}^{b}-X_{\text {ref }}^{a}\right| /\left|X_{\text {ref }}^{a}\right|
$$

onde $X_{\text {num }}$ é a solução numérica obtida com base no programa NASEN e $X_{r e f}$ é a solução de referência do problema, usualmente, igual a solução analítica do problema físico. Às vezes, por conta do desconhecimento dessa solução, utilizam-se resultados de testes numéricos/experimentais. erro médio percentual da amostragem total de dados é calculado: $\sum|\Delta| / n$, sendo $n$ a quantidade da amostragem.

\subsection{Viga sob base elástica submetida à ação de carregamentos externos}

Na presente seção, visa-se avaliar a eficiência do modelo computacional NASEN na solução do comportamento físico de vigas sob base elástica, conforme descrito pela Equação (1). A matriz de rigidez global do sistema linear é construída com base na matriz local de viga apresentada na Equação (10). O problema é modelado pelo elemento estrutural unidimensional (ver Figura 2). Sendo assim, estudam-se duas configurações associadas aos carregamentos e vinculações na viga, conforme mostrado na Figura 4. Em ambos os casos adota-se que o módulo de elasticidade é igual a 15 GPa e a seção transversal retangular com base e altura igual a 200 e $150 \mathrm{~mm}$, respectivamente. 
O primeiro caso é definido por uma viga biapoiada de comprimento igual a $8 \mathrm{~m}$ sob ação de uma carga distribuída de 100 kN/m e uma solicitação axial de tração de 1000 kN (ver Figura44a). Para este caso, busca-se extrair as informações do problema com relação ao campo de deslocamento e de inclinação da viga em função do comprimento da estrutura.

Figura 4 - Viga de fundação (a) biapoiada sujeita a uma carga transversal distribuída e horizontal de tração, e (b) com extremidades livres sujeita ao carregamento simétrico.

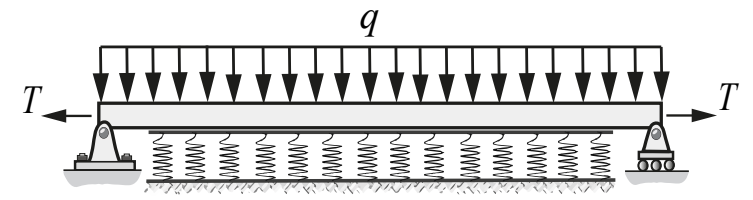

(a)

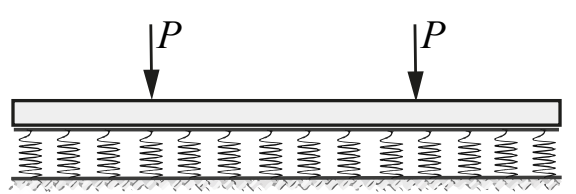

(b)

Fonte: Elaboração dos autores (2020).

A resposta numérica obtida para o campo de deslocamento da viga é comparada com solução analítica do problema, encontrada na literatura clássica da área (vide Coduto, Kitch e Yeung (2001) e Melerski (2006)). Nota-se na Figura 5a que as curvas obtidas para solução numérica e exata apresentam boa concordância, quantitativamente, para $k_{0}=40$, o erro médio percentual (ver Equação (13)) calculado entre a solução analítica e a numérica (NASEN), fornece um valor de aproximadamente $4,01 \cdot 10^{-8} \%$. A Figura 5 b mostra o campo de inclinação ao longo do comprimento da viga assumindo diferentes valores de coeficientes de proporcionalidade da base elástica, verificando que quando maior a rigidez da base elástica resulta-se em menores rotações na viga.

Figura 5 - (a) Campo de deslocamento e (b) de rotação da viga com carga transversal e axial.

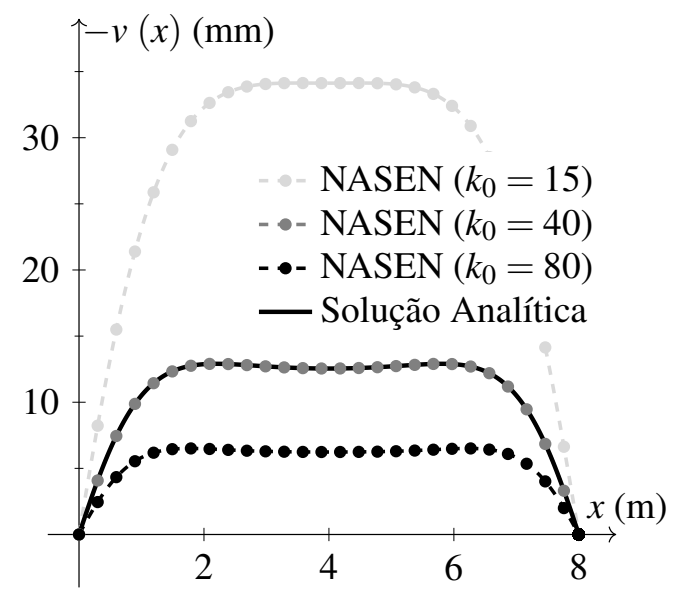

(a)

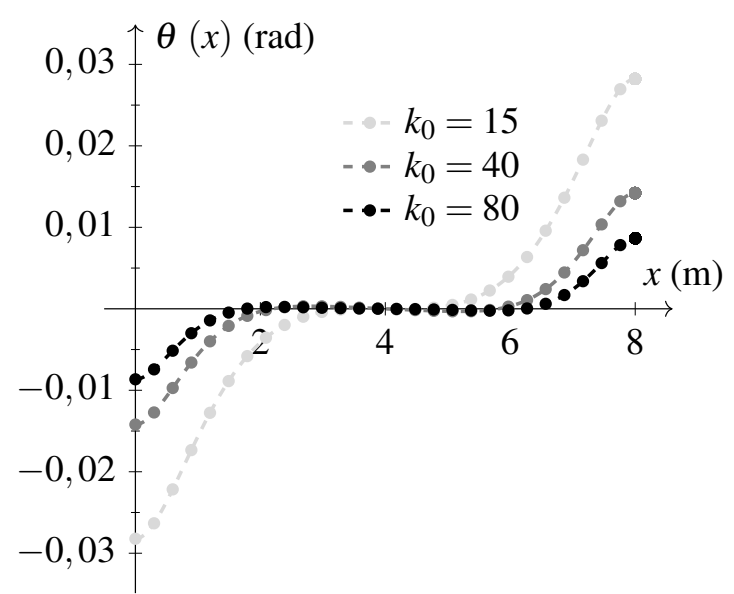

(b)

Fonte: Elaboração dos autores (2020). 
O segundo caso consiste em uma viga com suas extremidades livres, ou seja, não existe nenhuma restrição de movimento, permitindo deslocamentos verticais, horizontais e rotacionais. $\mathrm{O}$ comprimento da viga é igual a $10 \mathrm{~m}$ e o módulo da carga pontual vertical é 125 kN (ver Figura 4p). Adota-se também um coeficiente de proporcionalidade da base elástica igual a $100 \mathrm{kN} / \mathrm{m}^{2} / \mathrm{mm}$.

Figura 6-(a) Campo de deslocamento e (b) de momento fletor da viga com extremidades livres submetida ao carregamento concentrado simétrico.

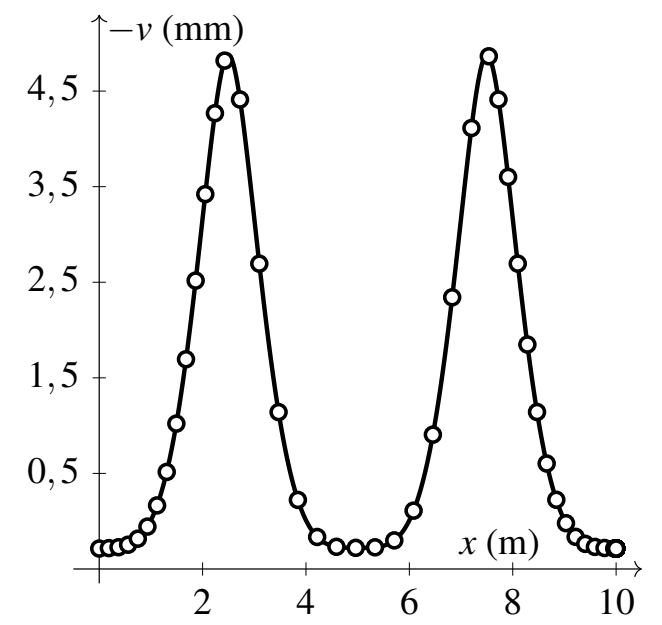

(a)

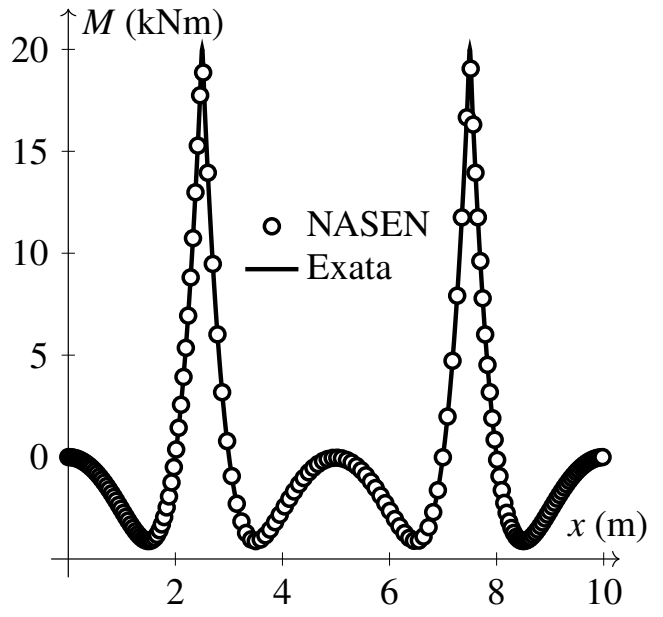

(b)

Fonte: Elaboração dos autores (2020).

Na Figura 6, apresenta-se uma comparação entre os resultados numéricos e analíticos. Pode-se verificar a boa semelhança entre os resultados, atingindo um erro médio percentual de $1,81 \cdot 10^{-6} \%$ e de $1,26 \cdot 10^{-1} \%$ em relação ao comportamento do campo de deslocamento $(v)$ e de momento fletor $(M)$ da viga, respectivamente. Observa-se que em razão do decaimento da ordem das funções de interpolação, o erro médio associado ao comportamento do momento fletor é maior em comparação ao medido com o campo de deslocamento.

Adicionamente, com objetivo de verificar o desempenho numérico do módulo computacional, apresenta-se na Tabela 1 os resultados do programa NASEN, utilizando o método de elementos finitos e de diferenças finitas para níveis de malha diferentes. Os valores numéricos do MDF são obtidos com um código autoral, desenvolvido em MATLAB, utilizando o esquema de diferenças centrais para as aproximações dos operadores diferenciais presentes na equação da viga (ver Equação (1)), conforme pode ser visto em Neves (2020). Os valores do deslocamento e do momento fletor são medidos em $x=2,5 \mathrm{~m}$ em relação à extremidade esquerda da viga, onde se tem uma carga concentrada aplicada. Nota-se que ambos os métodos apresentam bons resultados, contudo, para o deslocamento, a convergência do MEF é rápida em comparação ao MDF, apresentando baixos 
níveis de erro para malhas menos refinadas. Em contrapartida, quando se avalia o momento fletor, mesmo que o MEF apresenta-se erros percentuais menores em relação ao MDF, ambos os métodos apresentam um comportamento parelho em níveis de ordem de grandeza, atingindo no fim o valor esperado.

Tabela 1 - Comparação de resultados obtidos via método dos elementos finitos e diferenças finitas para deslocamento e momento fletor em $x=2,5 \mathrm{~m}$.

\begin{tabular}{|c|c|c|c|c|c|c|c|c|}
\hline \multirow{2}{*}{ NNós } & \multicolumn{4}{|c|}{ Deslocamento (mm) } & \multicolumn{4}{|c|}{ Momento Fletor (kNm) } \\
\hline & MEF $^{\top}$ & MDF $^{2}$ & $\Delta_{0}^{1}(\%)$ & $\Delta_{0}^{2}(\%)$ & MEF $^{1}$ & MDF $^{2}$ & $\Delta_{0}^{1}(\%)$ & $\Delta_{0}^{2}(\%)$ \\
\hline 7 & 4,1324 & 3,6520 & $1,53 \mathrm{E}+01$ & $2,52 \mathrm{E}+01$ & 13,3106 & 5,4450 & $3,35 \mathrm{E}+01$ & $7,28 \mathrm{E}+01$ \\
\hline 13 & 4,7519 & 5,4688 & $2,62 \mathrm{E}+00$ & $1,21 \mathrm{E}+01$ & 17,2869 & 10,4196 & $1,36 \mathrm{E}+01$ & $4,79 E+01$ \\
\hline 19 & 4,8505 & 5,4891 & $6,03 E-01$ & $1,25 \mathrm{E}+01$ & 18,6763 & 15,6109 & $6,68 \mathrm{E}+00$ & $2,20 E+01$ \\
\hline 31 & 4,8760 & 5,1735 & 8,13E-02 & $6,02 E+00$ & 19,5133 & 18,5505 & $2,50 \mathrm{E}+00$ & $7,31 E+00$ \\
\hline 43 & 4,8789 & 5,0393 & 2,13E-02 & $3,27 \mathrm{E}+00$ & 19,7557 & 19,2964 & $1,28 \mathrm{E}+00$ & $3,58 \mathrm{E}+00$ \\
\hline 61 & 4,8797 & 4,9601 & 5,13E-03 & $1,64 \mathrm{E}+00$ & 19,8862 & 19,6668 & $6,32 \mathrm{E}-01$ & $1,73 E+00$ \\
\hline 91 & 4,8799 & 4,9162 & 1,02E-03 & 7,44E-01 & 19,9563 & 19,8640 & 2,82E-01 & $7,43 E-01$ \\
\hline 247 & 4,8799 & 4,8848 & 1,82E-05 & $1,00 \mathrm{E}-01$ & 20,0052 & 19,9937 & 3,78E-02 & $9,50 \mathrm{E}-02$ \\
\hline Exata $^{0}$ & \multicolumn{2}{|c|}{4,8799} & \multicolumn{6}{|c|}{20,0127} \\
\hline
\end{tabular}

Fonte: Dados da pesquisa (2020).

4.2 Placa delgada submetida a um carregamento distribuído

Nesta seção, visa-se estudar, numericamente, o comportamento de uma placa delgada sob ação de carga externa (ver Equação (2)). A matriz de rigidez global de placa presente no sistema algébrico, apresentado na Equação (6), é definida pelo assemblamento da matriz local de cada elemento finito da malha, seguindo a Equação (11. Sendo assim, a Figura 7a apresenta uma placa fina retangular sujeita a uma carga distribuída uniformemente. Em termos de condições de contorno, todas as bordas da placa são engastadas, ou seja, os movimentos de rotação $\left(\theta_{x}\right.$ e $\left.\theta_{y}\right)$ e de deslocamento $(w)$ são restringidos.

Para este problema define-se alguns parâmetros: as dimensões da placa são iguais 200×400 $\mathrm{cm}$ e a espessura de $3,0 \mathrm{~cm}$, o módulo de elasticidade é igual a $21000 \mathrm{kN} / \mathrm{cm}^{2}$ e a intensidade da carga distribuída aplicada é igual a $2 \cdot 10^{-4} \mathrm{kN} / \mathrm{cm}^{2}$. Em termos computacionais realiza-se uma discretização do domínio analisado, usando elementos finitos regulares de quatro nós, as arestas da placa são subdivididas 32 intervalos, totalizando 1024 elementos finitos (ver Figura7p). 
Figura 7 - Placa retangular totalmente engastada sujeita ao carregamento distribuído uniforme.

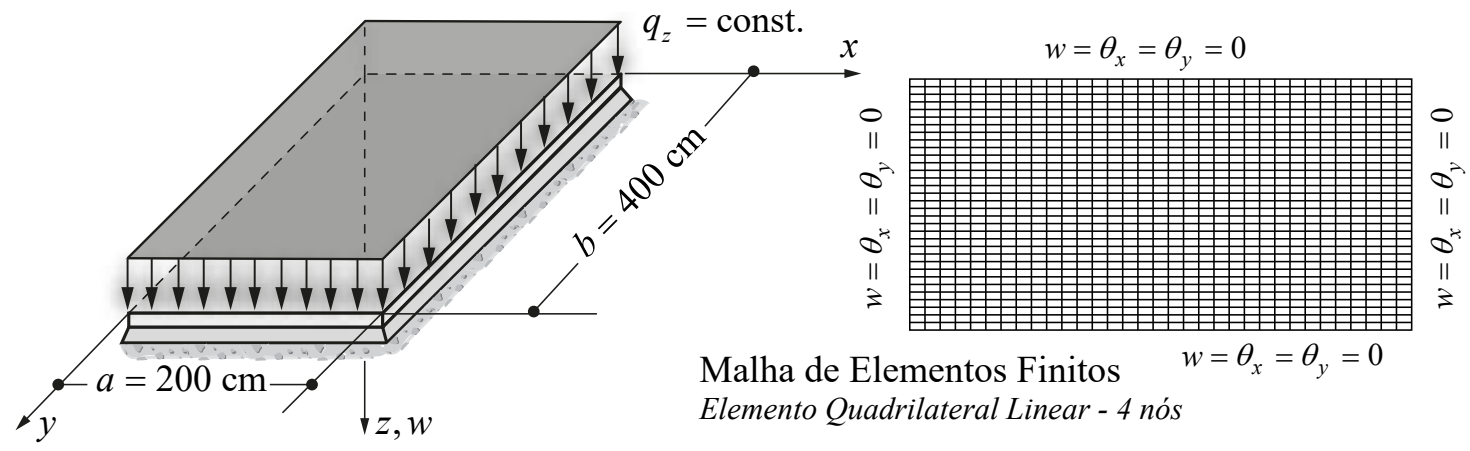

(a)

(b)

Fonte: Elaboração dos autores (2020).

Na Tabela 2, exibe-se os valores de deslocamento e momento no meio-vão da placa. Primeiramente, adota-se o coeficiente de Poisson igual a 0,2 e são consideradas como soluções de referência as tabelas de cálculo de Bares e Canals (1970) e Czerny (1976), bem como os dados numéricos fornecidos no trabalho Leal (2015), baseado no MEF. Quando se compara os dados utilizando o programa NASEN, observa-se uma semelhança nos valores encontrados. Com base na métrica de erro $(\Delta)$ definida na Equação [13, nota-se que as diferenças percentuais de resultados aparentes provenientes dos autores clássicos, deve-se ao fato que eles utilizam métodos e aproximações diferentes para a construção das respectivas tabelas de cálculo, fundamentados em métodos baseados em séries, aproximações de diferenças finitas ou também por meio da teoria da elasticidade.

Tabela 2 - Comparação dos valores obtidos para deslocamentos e momentos no meio do vão da placa em relação aos resultados da literatura.

\begin{tabular}{llccc}
\hline & & $w \mathbf{( m m})$ & $\mathbf{M}_{x x} \mathbf{( k N m )}$ & $\mathbf{M}_{y y}(\mathbf{k N m})$ \\
\hline \multirow{5}{*}{$v=0,2$} & NASEN $^{0}$ & $-1,649 \mathrm{E}-02$ & $3,263 \mathrm{E}-01$ & $9,438 \mathrm{E}-02$ \\
& Leal (2015) $^{1}$ & $-1,648 \mathrm{E}-02$ & $3,269 \mathrm{E}-01$ & $9,444 \mathrm{E}-02$ \\
& $\left|\Delta_{0}^{1}\right|(\%)$ & $5,295 \mathrm{E}-02$ & $1,763 \mathrm{E}-01$ & $6,860 \mathrm{E}-02$ \\
& Bares $^{2}$ & - & $3,256 \mathrm{E}-01$ & $9,280 \mathrm{E}-02$ \\
& $\left|\Delta_{0}^{2}\right|(\%)$ & - & $2,173 \mathrm{E}-01$ & $1,698 \mathrm{E}+00$ \\
& Czérny $^{3}$ & $-1,645 \mathrm{E}-02$ & $3,333 \mathrm{E}-01$ & $1,401 \mathrm{E}-01$ \\
& $\left|\Delta_{0}^{3}\right|(\%)$ & $2,332 \mathrm{E}-01$ & $2,108 \mathrm{E}+00$ & $3,264 \mathrm{E}+01$ \\
\hline \multirow{4}{*}{$v=0,3$} & NASEN $^{0}$ & $-1,562 \mathrm{E}-02$ & $3,293 \mathrm{E}-01$ & $1,264 \mathrm{E}-01$ \\
& Timoshenko $^{1}$ & $-1,565 \mathrm{E}-02$ & $3,296 \mathrm{E}-01$ & $1,264 \mathrm{E}-01$ \\
& $\left|\Delta_{0}^{1}\right|(\%)$ & $2,179 \mathrm{E}-01$ & $9,517 \mathrm{E}-02$ & $2,962 \mathrm{E}-02$ \\
\hline
\end{tabular}

Fonte: Dados da pesquisa (2020). 
Para a faixa de resultados associados ao coeficiente de Poisson igual a 0,3, adota-se como referência a solução obtida em Timoshenko e Woinowsky-Krieger (1959). Veja que neste cenário os valores encontrados para deslocamento e momento exibem um bom ajuste associados a erros percentuais abaixo de $0,5 \%$. Em problemas bidimensionais, a visualização de resultados é um fator importante a fim de verificar o comportamento da solução numérica obtida em cada ponto do domínio. Desta maneira, a Figura 8 a-b apresenta o campo de deslocamento vertical na placa e o momento fletor na direção $x$, onde pode-se notar que a flecha é nula nas bordas devido o engastamento e é máxima no centro da placa. Veja que ao longo da linha horizontal central, na extremidade e no centro da placa, existe a presença de momentos negativos e positivos, respectivamente.

Figura 8 - Distribuição bidimensional do (a) campo de deslocamento $w(x, y)$, do momento fletor (b) $M_{x x}(x, y)$, (c) $M_{y y}(x, y)$ e do (d) momento torsor $M_{x y}(x, y)$ da placa totalmente engastada.

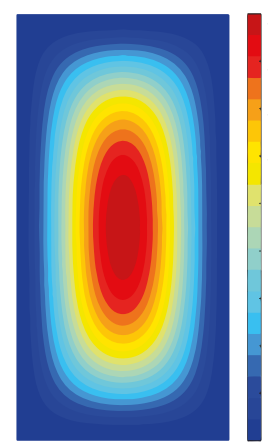

(a)

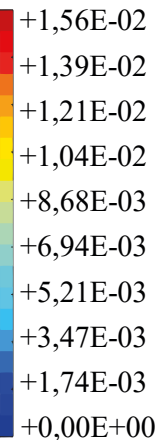

$+0,00 \mathrm{E}+00$

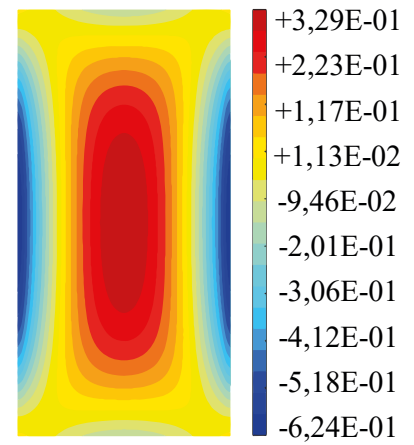

(b)

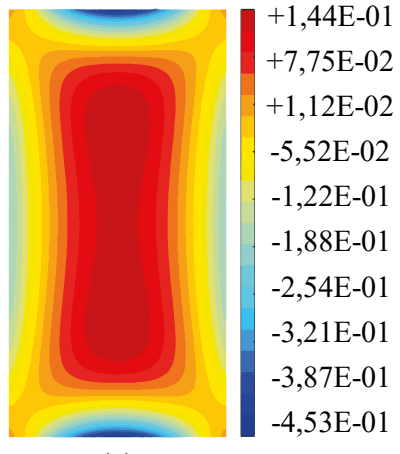

(c)

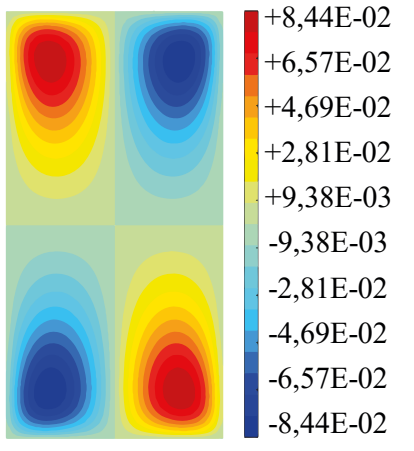

(d)

Fonte: Elaboração dos autores (2020).

Complementando a exposição gráfica dos resultados, tem-se ainda a apresentação do momento fletor na direção y e o momento torsor, conforme posto na Figura $8 \mathrm{c}$-d. Em relação ao eixo $y$, nota-se um comportamento inverso em relação ao momento na direção $x$, sendo que os momentos negativos estão localizados na borda superior e inferior da placa. Ressalta-se também que os cálculos dos momentos são baseados nas variáveis primais do problema. Esse fato acarreta que os momentos dependem da qualidade dos resultados obtidos para os deslocamentos em cada nó da placa.

\subsubsection{Viga engasta livre sob ação de uma carga concentrada}

Dentro da seara de problemas de estado plano de tensão (ver Equação (3)), estuda-se uma viga isostática engastada-livre de comprimento $L$, altura $h$ e espessura $t$, sujeita a uma carga vertical concentrada aplicada na extremidade livre, conforme ilustra a Figura 9a. A matriz de rigidez global é descrita pelo assemblamento das matrizes locais, conforme o padrão de formação apresentado na 
Equação (12). Emprega-se os seguintes dados e propriedades: a carga tem módulo igual a $4000 \mathrm{~N}$, momento de inércia da seção transversal é $1 \times 10^{-5} \mathrm{~m}^{4}$, as dimensões da viga são $100 \times 10 \times 12 \mathrm{~cm}$ e o módulo de elasticidade do material é $200 \mathrm{GPa}$.

Analisa-se nesse problema o deslocamento da extremidade em balanço e a tensão na viga. Os resultados obtidos pelo programa NASEN são comparados com solução analítica via teoria clássica de viga baseada nos princípios elementares da resistência dos materiais, conforme visto em detalhe em literaturas clássicas, como Hibbeler (2010) e Timoshenko (1979).

Figura 9 - (a) Modelo estrutural da viga engastada livre sob ação da carga concentrada e (b) distribuição de tensão normal na direção $x$.

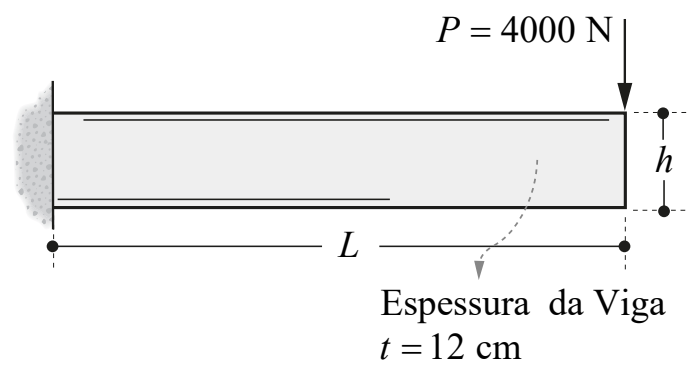

(a)

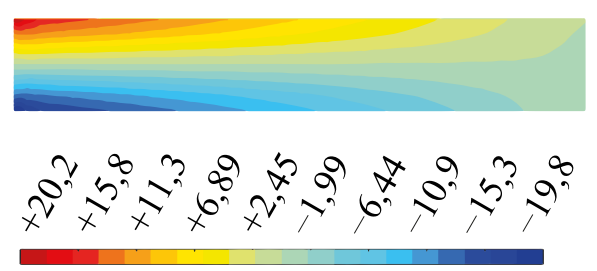

(b) $\sigma_{x x}[\mathrm{MPa}]$

Fonte: Elaboração dos autores (2020).

A Tabela 3 apresenta os valores encontrados em função do tipo de elemento finito utilizado na simulação, refinamento da malha e número de graus de liberdade do sistema. Observa-se que o elemento triangular linear de três nós (T3) exibe os maiores erros em comparação com os demais tipos de elementos, porém, solução tende a melhorar os resultados com o refinamento da malha. Ressalta-se também que esse tipo de elemento é útil para modelar geometrias mais complexas. Além disso, a integração numérica pode ser realizada com somente um ponto de Gauss, caracterizando um processamento mais acelerado.

O elemento de quatro nós (Q4) apresenta um bom comportamento, ao passo que para um nível de refinamento relativamente baixo, os resultados são parelhos aos elementos finitos de altaordem. Os elementos quadriláteros de oito (Q8) e nove (Q9) nós apresentam baixos níveis de erro, contudo, revelam também um rápido crescimento no número de graus de liberdade do sistema, demandando um maior custo computacional. 
Tabela 3 - Valores de deslocamento e de tensão para diferentes tipos de elementos finitos.

\begin{tabular}{ccccccc}
\hline $\begin{array}{c}\text { Tipo de } \\
\text { Elemento }\end{array}$ & Malha & $\begin{array}{c}\mathbf{N}^{\circ} \text { Graus } \\
\text { de Liberdade }\end{array}$ & $v(\mathbf{m})^{1}$ & $\left|\Delta_{0}^{1}\right| \mathbf{( \% )}$ & $\sigma(\mathbf{M P a})^{2}$ & $\left|\Delta_{0}^{2}\right|(\%)$ \\
\hline \multirow{2}{*}{ T3 } & 364 & 446 & $-5,42 \mathrm{E}-04$ & $1,86 \mathrm{E}+01$ & 15,64 & $2,18 \mathrm{E}+01$ \\
& 1002 & 1164 & $-6,34 \mathrm{E}-04$ & $4,86 \mathrm{E}+00$ & 20,45 & $2,27 \mathrm{E}+00$ \\
\hline \multirow{2}{*}{$\mathbf{Q 4}$} & 40 & 126 & $-5,97 \mathrm{E}-04$ & $1,05 \mathrm{E}+01$ & 18,62 & $6,90 \mathrm{E}+00$ \\
& 250 & 612 & $-6,58 \mathrm{E}-04$ & $1,30 \mathrm{E}+00$ & 21,37 & $6,84 \mathrm{E}+00$ \\
\hline \multirow{2}{*}{ Q8 } & 25 & 192 & $-6,71 \mathrm{E}-04$ & $6,45 \mathrm{E}-01$ & 19,70 & $1,51 \mathrm{E}+00$ \\
& 40 & 330 & $-6,71 \mathrm{E}-04$ & $5,70 \mathrm{E}-01$ & 19,87 & $6,50 \mathrm{E}-01$ \\
\hline \multirow{2}{*}{ Q9 } & 10 & 126 & $-6,77 \mathrm{E}-04$ & $1,49 \mathrm{E}+00$ & 19,00 & $5,00 \mathrm{E}+00$ \\
& 36 & 338 & $-6,72 \mathrm{E}-04$ & $7,20 \mathrm{E}-01$ & 19,73 & $1,34 \mathrm{E}+00$ \\
\hline \multicolumn{7}{l}{ Teoria Clássica de Viga } \\
\hline
\end{tabular}

Fonte: Dados da pesquisa (2020).

Na Figura 10, mostra-se o perfil de tensão normal e cisalhante na extremidade fixa, medido ao longo da altura da viga. O conhecimento desses perfis de tensões é importante para engenharia a fim de auxiliar na investigação dos efeitos internos no elemento estrutural, por exemplo, nota-se a visível diferença da ordem de grandeza entre as tensões normais $\sigma_{x x}$ e $\sigma_{y y}$ (ver Figuras 10a-b) - explicando o motivo que em diversas aplicações a tensão na direção y não é considerada nos cálculos de engenharia. Observa-se ainda que a tensão normal na direção $x$, obtida numericamente com base no modelo da elasticidade plana, apresenta boa aderência quando comparada com a fórmula proveniente da teoria clássica de viga (TCV), variando linearmente em relação à altura da viga, conforme apresentado em Hibbeler (2010) e Timoshenko (1979). Além disso, o perfil de tensão de cisalhamento da viga apresenta um comportamento parabólico, atingido o valor máximo no centro da seção transversal (ver Figura 10k). Adicionalmente, a Figura $9 \mathrm{p}$ mostra a distribuição de tensão normal, onde é possível verificar as regiões tracionadas (fibras superiores) e comprimidas (fibras inferiores). Além disso, a tensão é nula extremidade livre, uma vez que não atua momento fletor nesse ponto. 
Figura 10 - Perfil de tensão na (a) direção $x$ e (b) direção $y$, e de (c) tensão cisalhante.

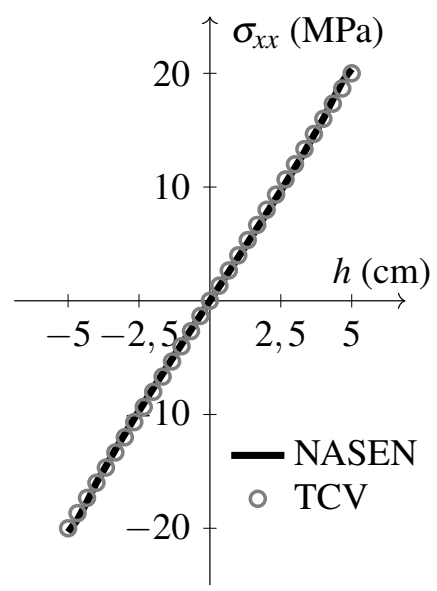

(a)

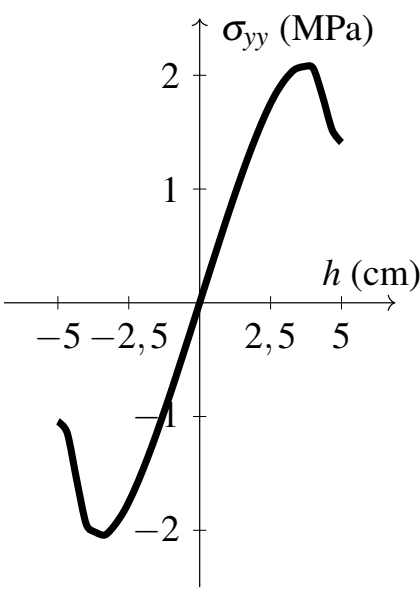

(b)

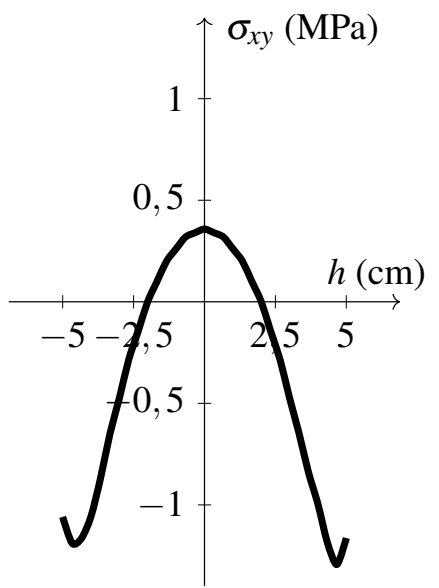

(c)

Fonte: Elaboração dos autores (2020).

\subsubsection{Chapa tracionada com orifício circular}

O próximo estudo de caso é constituído por uma chapa sob ação de uma tração uniforme, conforme mostra a Figura 11a. Esse problema busca avaliar o desempenho do programa NASEN para determinação do campo de tensão efetiva ou ruptura (NEVES; PINHEIRO, 2019). Neste contexto, considera-se o critério de ruptura baseado na teoria da energia de distorção máxima ou usualmente conhecido como critério de falha de von Mises (ver Equação (4)), para maiores detalhes e aprofundamentos sobre criterios de falhas, vide Popov (1978) e Timoshenko (1979).

Figura 11 - (a) Geometria e condições de contorno da chapa sob ação da tração uniforme e (b) distribuição bidimensional de tensão efetiva.

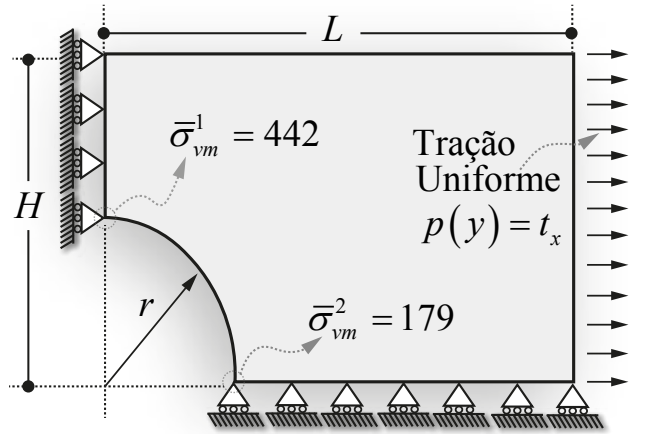

(a)

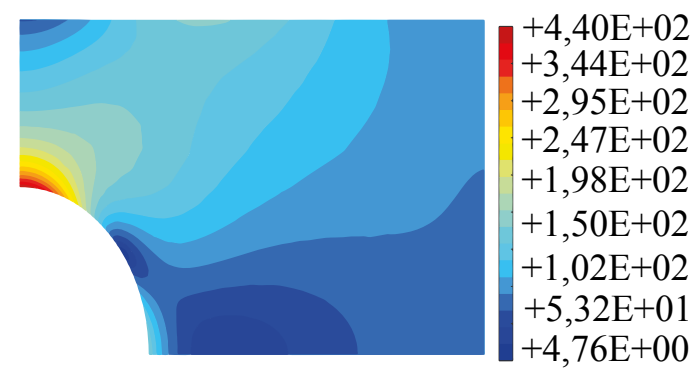

(b)

Fonte: Elaboração dos autores (2020).

Assume-se que o comprimento da chapa é igual a $L=18$, a altura de $H=10$, o raio da cavidade circular igual a $r=5$ e a espessura é considerada unitária. A carga aplicada na borda 
direita da chapa é $p=100$, o módulo de elasticidade é adotado igual a $72 \cdot 10^{9}$ e o coeficiente de Poisson é nulo.

Em relação à simulação computacional, a Figura 12 apresenta o domínio discretizado em função de cada tipo de elemento utilizado para solução do problema. Para cada tipo de elemento finito são realizados três simulações com um fator de refinamento em cada aresta da chapa de 5, 10 e 15 divisões. Os resultados numéricos do programa NASEN são comparados com a solução exata do problema, sendo essa obtida em Payen e Bathe (2011). Os valores analíticos da tensão de von Mises $\bar{\sigma}_{v m}^{1}$ e $\bar{\sigma}_{v m}^{2}$, medidos na extremidade superior (ponto 1) e direita (ponto 2) do orifício circular, são obtidos por meio dos procedimentos de solução via teoria da elasticidade.

Figura 12 - Malhas de elementos finitos utilizadas nas simulações computacionais.
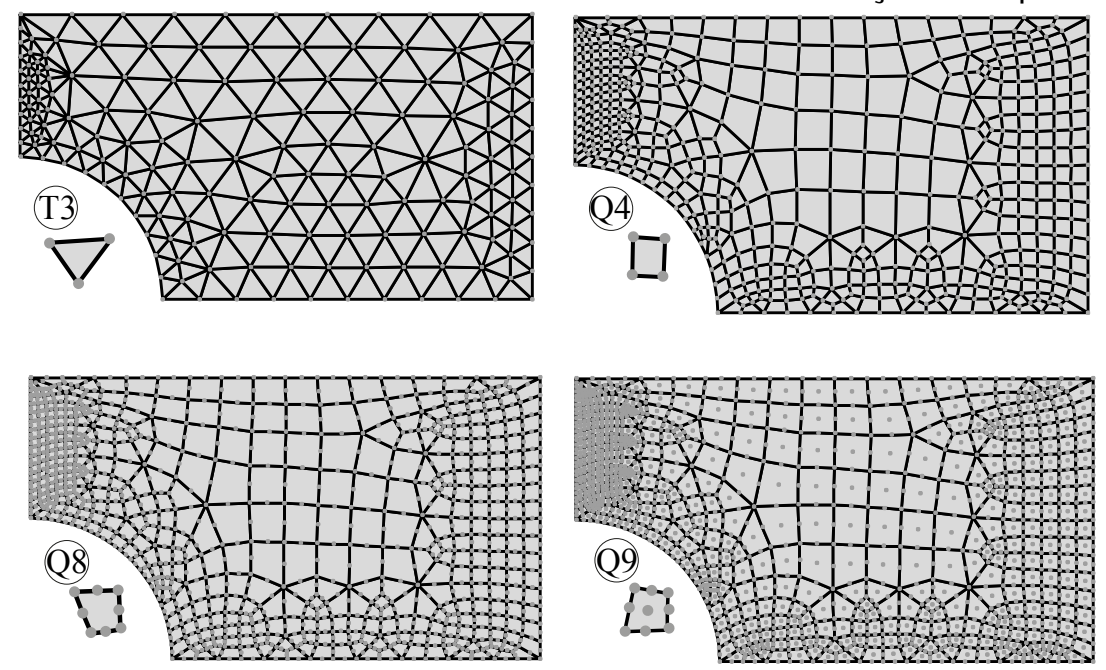

Fonte: Elaboração dos autores (2020).

$\mathrm{Na}$ Tabela 4, apresenta-se os valores obtidos das tensões von Mises em cada simulação associada ao tipo de elemento finito testado. Primeiramente, deve-se frisar que o cálculo da tensão de von Mises carrega ao longo do processamento inúmeras aproximações que dependem das tensões planas e sendo essas determinadas a partir do campo de deslocamento.

Em síntese, todos os elementos apresentaram bons resultados quando comparados com a solução de referência. Os elementos do tipo quadráticos de oito e nove nós apresentam melhores resultados, justificado pela eficiência no processo de suavização de tensão, em contrapartida, tais tipos de elementos apresentam um número maior de graus de liberdade do sistema, exigindo um maior custo computacional. Adicionalmente, na Figura 11p apresenta-se o campo bidimensional de tensão efetiva de ruptura de von Mises, onde ocorre uma concentração de tensão próxima ao canto superior do orifício (ponto 1), sendo uma região de possível ruptura do material. 
Tabela 4 - Valores numéricos obtidos para as tensões von Mises nos pontos 1 e 2 da chapa com orifício.

\begin{tabular}{|c|c|c|c|c|c|}
\hline $\begin{array}{l}\text { Tipo de } \\
\text { Elemento }\end{array}$ & Malha & $\sigma_{v m}^{1}$ & $\left|\Delta_{0}^{1}\right|(\%)$ & $\sigma_{v m}^{2}$ & $\left|\Delta_{0}^{2}\right|(\%)$ \\
\hline \multirow{3}{*}{ T3 } & 280 & $3,18 \mathrm{E}+02$ & $2,81 \mathrm{E}+01$ & $8,43 \mathrm{E}+01$ & $5,29 \mathrm{E}+01$ \\
\hline & 413 & $3,83 E+02$ & $1,30 E+01$ & $1,58 \mathrm{E}+02$ & $1,17 E+01$ \\
\hline & 1330 & 4,27E+02 & $3,40 \mathrm{E}+00$ & $1,69 \mathrm{E}+02$ & $5,59 E+00$ \\
\hline \multirow{3}{*}{ Q4 } & 103 & $3,64 \mathrm{E}+02$ & $1,76 \mathrm{E}+01$ & $1,10 \mathrm{E}+02$ & $3,85 \mathrm{E}+01$ \\
\hline & 442 & $4,26 \mathrm{E}+02$ & $3,61 E+00$ & $1,63 E+02$ & $8,94 \mathrm{E}+00$ \\
\hline & 1065 & $4,36 \mathrm{E}+02$ & $1,35 E+00$ & $1,72 E+02$ & $3,91 E+00$ \\
\hline \multirow{3}{*}{ Q8 } & 103 & $4,20 \mathrm{E}+02$ & $4,98 \mathrm{E}+00$ & $1,72 \mathrm{E}+02$ & $3,91 \mathrm{E}+00$ \\
\hline & 442 & $4,37 E+02$ & $1,13 E+00$ & $1,67 E+02$ & $6,70 E+00$ \\
\hline & 1065 & $4,41 \mathrm{E}+02$ & $2,26 \mathrm{E}-01$ & $1,75 \mathrm{E}+02$ & $2,23 E+00$ \\
\hline \multirow{3}{*}{ Q9 } & 103 & $4,22 \mathrm{E}+02$ & $4,52 \mathrm{E}+00$ & $1,74 \mathrm{E}+02$ & $2,79 \mathrm{E}+00$ \\
\hline & 442 & $4,38 \mathrm{E}+02$ & $1,02 E+00$ & $1,75 E+02$ & $2,23 E+00$ \\
\hline & 1065 & 4,41E+02 & $1,81 \mathrm{E}-01$ & $1,76 \mathrm{E}+02$ & $1,79 E+00$ \\
\hline \multicolumn{2}{|c|}{ Solução Exata ${ }^{0}$} & $4,42 \mathrm{E}+02$ & & 1,79E+02 & \\
\hline
\end{tabular}

Fonte: Dados da pesquisa (2020).

\section{Considerações Finais}

O presente artigo científico teve como objetivo apresentar um módulo computacional específico desenvolvido para análise linear de elementos estruturais com base na teoria de modelos unidimensionais, de placa finas e da elasticidade plana. Os procedimentos de cálculo têm como base as premissas do método dos elementos finitos e a implementação computacional estruturada é realizada em ambiente MATLAB.

A experimentação numérica é direcionada por casos testes conhecidos na literatura, visando realizar uma validação dos resultados e uma verificação da performance do programa para diferentes configurações. Desta forma, em todos os casos estudados e investigados com programa NASEN, os resultados obtidos são satisfatórios com um comportamento coerente fisicamente. Em linhas gerais, o programa computacional desenvolvido é dito validado e pode ser aplicada para auxiliar em análises iniciais e pré-cálculos em projetos de estruturas. Para trabalhos futuros, busca-se considerar diferentes efeitos físicos importantes para problemas de engenharia e expandir as aplicabilidades do programa para outras áreas de conhecimento.

\section{Referências}

ARGYRIS, J. H.; BONI, B.; HINDENLANG, U.; KLEIBER, M. Finite element analysis of two-and three-dimensional elasto-plastic frames-the natural approach. Computer Methods in Applied 
Mechanics and Engineering, v. 35, n. 2, p. 221-248, 1982. DOI: https://doi.org/10.1016/0045-7825(82)90135-9.

BARES, R.; CANALS, J. M. Tablas para el cálculo de placas y vigas pared. Barcelona: Gustavo Gili, 1970.

CARVALHO, R. C.; FIGUEIREDO FILHO, J. R. Cálculo e detalhamento de estruturas usuais de concreto armado: segundo a NBR 6118: 2014. São Carlos: EdUFSCar, 2014.

CODUTO, D. P.; KITCH, W. A.; YEUNG, M. R. Foundation design: principles and practices. Upper Saddle River: Prentice Hall, 2001.

CZERNY, C. Beton-kalender. Berlim: Wilhelm Ernst \& Sohn, 1976.

EULER, L. De Curvis Elasticis, Additamentum I to his Methodus Inveniendi Lineas Curvas Maximi Minimive Proprietate Gaudentes. Lausanne and Geneva: Springer Science \& Business Media, 1744.

FRANSSEN, J. M. SAFIR: A thermal/structural program for modeling structures under fire. Engineering Journal, v. 42, n. 3, p. 143-158, 2005.

GEUZAINE, C.; REMACLE, J.-F. Gmsh: A 3-d finite element mesh generator with built-in pre-and post-processing facilities. International Journal for Numerical Methods in Engineering, v. 79, n. 11, p. 1309-1331, 2009. DOl: https://doi.org/10.1002/nme.2579.

HIBBELER, R. C. Resistência dos materiais. São Paulo: Pearson Prentice Hall, 2010.

LEAL, C. E. F. Formulação do método dos elementos finitos para a análise elástica linear de placas delgadas. Orientador: Leandro Waidemam. 2015. 136 f. Trabalho de Conclusão de Curso (Engenharia Civil) - Departamento Acadêmico de Construção Civil, Universidade Tecnológica Federal do Paraná, Campo Mourão, 2015.

LOPES, A. M. G. A versatile software tool for the numerical simulation of fluid flow and heat transfer in simple geometries. Computer Applications in Engineering Education, v. 18, n. 1, p. 14-27, 2010. DOI: https://doi.org/10.1002/cae.20230.

MALISKA, C. R. Transferência de calor e mecânica dos fluidos computacional. Barueri: Grupo Gen-LTC, 2017.

MARTHA, L. F. FTOOL: Um programa gráfico-interativo para ensino de comportamento de estruturas. Versão Educacional 2.11. Rio de Janeiro: PUC, ago. 2002.

MATLAB. Version 7.10.0 (R2010a). Natick, Massachusetts: The MathWorks Inc, 2010.

MAXIMIANO, D. P.; SILVEIRA, R. A.; SILVA, A. R.; GONÇALVES, P. B. An efficient strategy for solving structural nonlinear equations by combining the orthogonal residual method and normal flow technique. International Journal of Structural Stability and Dynamics, v. 19, n. 4, p. 1950039, 2019. DOI: https://doi.org/10.1142/S0219455419500391.

MELERSKI, E. S. Design analysis of beams, circular plates and cylindrical tanks on elastic foundations. London: Taylor \& Francis, 2006. 
NEVES, N. S. Modelo computacional avançado para análise de estruturas sob ação de gradientes térmicos. Orientador: Macksuel Soares de Azevedo. Coorientador: Rodrigo Silveira Camargo. 2019. 169 f. Dissertação (Mestrado em Engenharia Civil) - Programa de Pós-Graduação em Engenharia Civil, Universidade Federal do Espírito Santo, Vitória, 2019a.

NEVES, N. S. Introdução aos procedimentos de elementos finitos aplicados à Teoria de Vigas de Timoshenko. In: Engenharia no Século XXI, v. 9, 1. ed. Belo Horizonte: Poisson, p. 159-169, 2019b. DOl: https://doi.org/10.36229/978-85-7042-163-0.CAP.18.

NEVES, N. S. Uma introdução aos procedimentos numéricos do método diferenças finitas: um estudo do comportamento de vigas de fundação na engenharia civil. Revista Tecnologia, v. 41, n. 1, 2020. DOl: https://doi.org/10.5020/23180730.2020.10683.

NEVES, N. S.; PINHEIRO, V. P. Determinação do campo de tensão efetiva em um problema vetorial elástico-linear via método de elementos finitos. In: ENCONTRO CIENTÍFICO DE FÍSICA APLICADA, 10, Vitória, 2019. Blucher Physics Proceedings. São Paulo: Editora Blucher, v. 6, p. 85-87, 2019.

NEVES, N. S; SOUZA, R. M.; PINHEIRO, V. P. A importância dos modelos teóricos e técnicas numéricas na construção de um elo com a prática da engenharia estrutural. Revista Eixo, v. 7, n. 1, p. 26-35, 2018. DOI:https://doi.org/10.19123/eixo.v7i1.490.

PAYEN, D. J.; BATHE, K. J. The use of nodal point forces to improve element stresses. Computers \& Structures, v. 89, n. 5-6, p. 485-495, 2011. DOI: https://doi.org/10.1016/j.compstruc.2010.12.002.

PIERIN, I.; SILVA, V. P.; LA ROVERE, H. L. Thermal analysis of two-dimensional structures in fire. Revista IBRACON de Estruturas e Materiais, v. 8, n. 1, p. 25-36, 2015. DOI:

http://dx.doi.org/10.1590/S1983-41952015000100005.

PITANGUEIRA, R. L. S.; FONSECA, F. T.; FUINA, J. S.; CAMARA, L.; FERREIRA, R. L.; MOREIRA, R. N.; FONSECA, M. T. INSANE - Versão 2.0. In: CILAMCE, 27, Maceió, 2008. Anais... Maceió: UFAL, p. 1-20, 2008.

POPOV, E. P. Introdução à mecânica dos sólidos. São Paulo: Blucher, 1978.

RANGEL, R. L.; MARTHA, L. F. LESM: An object-oriented MATLAB program for structural analysis of linear element models. Computer Applications in Engineering Education, v. 27, n. 3, p. 553-571, 2019. DOl: https://doi.org/10.1002/cae.22097.

REDDY, J. N. An introduction to the finite element method. v. 27. New York: McGraw-Hill, 1993.

SILVA, A. R. D. D.; PRADO, Í. M.; SILVEIRA, R. A. D. M. CS-ASA: a new computational tool for advanced analysis of steel frames. Rem: Revista Escola de Minas, v. 66, n. 3, p. 281-288, 2013. DOI: https://doi.org/10.1590/S0370-44672013000300003.

TIMOSHENKO, S. Resistência dos Materiais. Rio de Janeiro: LTC, 1979.

TIMOSHENKO, S.; WOINOWSKY-KRIEGER, S. Theory of plates and shells. New York: McGraw-Hill, 1959. 
TORKAMANI, M. A.; SONMEZ, M.; CAO, J. Second-order elastic plane-frame analysis using finite-element method. Journal of Structural Engineering, v. 123, n. 9, p. 1225-1235, 1997. DOI: https://doi.org/10.1016/j.engstruct.2010.04.033.

WEST, H. H. Analysis of structures, an integration of classical and modern methods. New York: John Wiley \& Sons, 1989.

ZIEMIAN, R. D.; MCGUIRE, W. Modified tangent modulus approach, a contribution to plastic hinge analysis. Journal of Structural Engineering, v. 128, n. 10, p. 1301-1307, 2002. DOI: https://doi.org/10.1061/(ASCE) 0733-9445(2002)128:10(1301).

ZIENKIEWICZ, O. C.; TAYLOR, R. L. The finite element method for solid and structural mechanics. Elsevier, 2005. 